\title{
Comparison of Clinical and Radiologic Characteristics between Anthracofibrosis and Endobronchial Lung Cancer
}

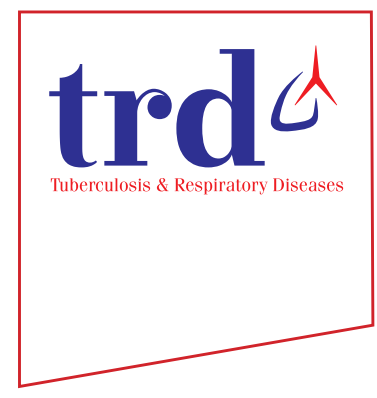

\author{
Seo Young Yun, M.D. ${ }^{(i)}$ and Tae Yun Park, M.D. \\ Division of Pulmonary and Critical Care Medicine, Department of Internal Medicine, Seoul Metropolitan Government Seoul \\ National University Boramae Medical Center, Seoul, Republic of Korea
}

Background: Endobronchial lung cancer (EBLC) and bronchial anthracofibrosis (BAF) share similar symptoms and radiological findings. The aim of this study was to describe clinical and radiological differences between BAF and EBLC, both of which were confirmed by bronchoscopy.

Methods: This was a retrospective study of patients with BAF or EBLC from 2008 to 2014. Data were derived from a bronchoscopy registry made since January 1, 2008. Clinical and radiological characteristics of both diseases were analyzed.

Results: Among 3,214 patients who underwent bronchoscopy, 167 and 117 patients were enrolled in BAF and EBLC groups, respectively. BAF occurred more predominantly in older non-smoking female patients with a higher chance of tuberculosis (38.3\%) than EBLC (6.0\%). Cough, sputum, and dyspnea were common symptoms reported for both groups. Bronchoscopic findings revealed that BAF lesions were more common in multiple lobar bronchi (85.0\%) or bilateral bronchi (73.7\%). Radiologic findings revealed that bronchial stenosis was the most commonly found lesion in both groups ( $49.1 \%$ and $78.6 \%$, respectively). Rates of peribronchial calcification and bronchial wall thickening were higher in the BAF group. The number of patients with lymph node calcification was also higher in the BAF group.

Conclusion: Results of this study demonstrated characteristics of clinical and radiologic findings of BAF and EBLC. Increasing the awareness of both diseases may help clinicians differentiate these two diseases from each other, thus avoiding unnecessary invasive diagnostic procedures.

Keywords: Bronchial Annthracofibrosis; Endobronchial Lung Cancer; Bronchoscopy

Address for correspondence: Tae Yun Park, M.D.

Division of Pulmonary and Critical Care Medicine, Department of Internal Medicine, Seoul Metropolitan Government Seoul National University Boramae Medical Center, 20 Boramae-ro 5-gil, Dongjak-gu, Seoul 07061, Republic of Korea

Phone: 82-2-870-3033, Fax: 82-2-831-2826

E-mail: cally0329@hanmail.net

Received: Dec. 5, 2020

Revised: Feb. 24, 2021

Accepted: May. 3, 2021

Published online: May. 13, 2021

(c) It is identical to the Creative Commons Attribution Non-Commercial License (http://creativecommons.org/licenses/by-nc/4.0/). The Korean Academy of Tuberculosis and Respiratory Diseases.

\section{Introduction}

Bronchial anthracofibrosis (BAF) is defined as bronchial narrowing with black pigmentation of the bronchial mucosa ${ }^{1}$. It is associated with chronic biomass exposure and active or old tuberculosis (TB). Although the actual prevalence of BAF remains unknown, several studies have reported that it is less than 5\% among patients who have undergone bronchosco$\mathrm{py}^{2,3}$. Although BAF is a rare disease, it is worthy of notice because it is often misdiagnosed as endobronchial lung cancer (EBLC $)^{4,5}$ due to the fact that these two diseases share several similarities. First, their radiologic similarities make diagnosis difficult. Common findings of BAF and TB include bronchial narrowing and obstruction that can be shown in endobronchial tumors. Second, most BAF patients are the elderly who usually complain of cough, sputum, and dyspnea. Patients with cancer also present these clinical features. Thus, it can be 
difficult to differentiate BAF from EBLC.

Bronchoscopy is one of the most detailed tests to diagnose BAF and EBLC. However, performing bronchoscopy in all suspected patients can be risky because most patients with $\mathrm{BAF}$ are the elderly. Therefore, more convenient and simpler diagnostic methods for characterizing BAF are needed. Today, computed tomography (CT) scans can be performed at a lower cost with reduced hazards. They can be used to detect characteristic radiologic features of BAF and EBLC. Characteristic CT features and clinical findings of both diseases can help us make better decisions and avoid unnecessary tests for BAF patients. Despite the necessity of a detailed study about these two diseases, studies that directly compare BAF and EBLC have not been reported yet. Therefore, the objective of this comparative study was to identify clinical and radiological features that could differentiate between BAF and EBLC, both of which were major causes of bronchial stenosis.

\section{Materials and Methods}

\section{Study design and patients}

This retrospective analysis was conducted on 284 patients with BAF or EBLC confirmed by bronchoscopy among 3,214 patients who underwent bronchoscopy between 2008 and 2014 at Seoul Metropolitan Government Seoul National University Boramae Medical Center Hospital in the Republic of Korea. Data were derived from a bronchoscopy registry created on January 1, 2008. BAF was defined as a bronchial narrowing with multiple dark pigmentations of the bronchial mucosa on the bronchoscopic view. EBLC was defined as a tumor involving the bronchial lumen on the bronchoscopic view and confirmed by bronchoscopic biopsy. Inclusion criteria for the study group were as follows: (1) patients older than 18 years, and (2) BAF or EBLC confirmed by two independent pulmonologists. Clinical characteristics including age, sex, body mass index, residence, smoking history, past medical history, pulmonary function test, and respiratory symptoms were evaluated. This study was approved by the Institutional Review Board (IRB) of Seoul National University Boramae Hospital (IRB No. 30-2020-247). The need for informed consent was waived due to the retrospective nature of this study.

\section{Bronchoscopic variables}

Common indications for bronchoscopy were as follows: possible TB $(\mathrm{n}=1,107,34.4 \%)$, possible lung cancer $(\mathrm{n}=873$, $27.2 \%)$, pneumonia $(\mathrm{n}=648,20.2 \%)$, hemoptysis $(\mathrm{n}=314,9.8 \%)$, interstitial lung disease $(\mathrm{n}=68,2.1 \%)$, non-TB mycobacterial infection ( $\mathrm{n}=44,1.4 \%)$, and unknown $(\mathrm{n}=160,5.0 \%)$. Before bronchoscopy, $2 \%$ lidocaine was used for local anesthesia (5 $\mathrm{mL}$ for gargling and four puffs of spray onto vocal cords). A bronchoscopic exam was performed by two pulmonologists (bronchoscopists). The location of the lesion, washing, and biopsy results were also evaluated. The location of relevant lesion was named as an involved bronchus according to lobar bronchial anatomy on bronchoscopic examination. Bronchoscopic biopsy was performed according to the pulmonologist's clinical judgment.

\section{Chest CT imaging and radiographic variables}

CT analysis included radiographic characteristics of bronchial trees (such as bronchial stenosis, atelectasis, peribronchial soft tissue infiltration or calcification) and lymph nodes (such as lymph node enlargement and location, number, and calcification of lymph nodes). On CT images, any lymph node with a short-axis diameter greater than $10 \mathrm{~mm}$ was reported as enlarged according to Response Evaluation Criteria in Solid Tumors (RECIST) v 1.1. We defined a lymph node containing calcification of any size as a calcified lymph node. Calcification of lymph nodes was categorized by size, extent, and pattern of calcification. The size of the calcified lymph node was measured unidimensionally (short-axis diameter) and classified into three groups (focal, capsular, and complete) according to a previous research. CT scans were reviewed retrospectively by a chest radiologist and a pulmonologist.

\section{Statistical analysis}

Values for continuous variables are reported as mean \pm standard deviation. Categorical variables are described as absolute frequencies and percentages. To compare two groups, $t$ test was used for continuous variables and chi-square test was used for categorical variables. All statistical analyses were performed using SPSS software version 18.0 for Windows (SPSS Inc., Chicago, IL, USA). A p-value of less than 0.05 was considered statically significant.

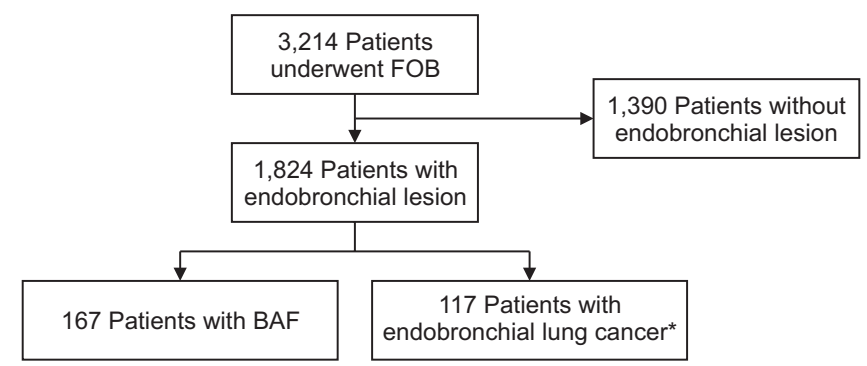

Figure 1. Scheme and flow showing the selection of study subjects from the bronchoscopy registry. FOB: fiberoptic bronchoscopy; BAF: bronchial anthracofibrosis. *Lung cancer lesion detected by FOB. 


\section{Results}

Among 3,214 patients who underwent bronchoscopy, finally 167 patients with BAF were enrolled for the BAF group and 117 patients with EBLC were enrolled for the EBLC group (Figure 1). Demographic and clinical characteristics of these patients are summarized in Table 1. Compared to the EBLC group, the BAF group had older patients (76.7 years vs. 68.2 years, $\mathrm{p}<0.05)$, higher proportion of females ( $67.7 \%$ vs. $15.4 \%$, $\mathrm{p}<0.05)$, and lower smoking rate ( $11.4 \%$ vs. $82.1 \%, \mathrm{p}<0.05)$. Patients with BAF were more likely to have active TB $(38.3 \%$ vs. $6.0 \%, \mathrm{p}<0.05)$. BAF patients showed better values of forced expiratory volume in 1 second $\left(\mathrm{FEV}_{1}\right)$ and forced vital capacity (FVC).

Table 2 shows main symptoms of BAF and EBLC. Common symptoms reported by patients were cough, sputum, and dyspnea in both groups. Meanwhile, the BAF group had lower percentages of blood-tinged sputum (2.4\% vs. $15.4 \%, \mathrm{p}<0.05)$, weight loss ( $5.4 \%$ vs. $11.1 \%$, p=0.078), and chest pain (3.0\% vs. $12.0 \%, \mathrm{p}<0.005)$ than the EBLC group.

Anatomical distributions of both diseases in the bronchial tree are shown in Table 3. The BAF group had the following distributions: right upper lobe (RUL), 47.9\%; right middle lobe (RML), 53.9\%; right lower lobe (RLL), 21.6\%; left upper lobe (LUL), 56.3\%; and left lower lobe (LLL), 24.6\%. The EBLC group had the following distributions: RUL, 37.6\%; RML, 12.0\%; RLL, 32.5\%; LUL, 51.4\%; and LLL, 17.1\%. Upper lobe predominance was observed in both groups. However, the rate of upper lobe involvement was much higher in the $\mathrm{BAF}$ group than in the EBLC group (146 [87.4\%] vs. 76 [65.0\%], $\mathrm{p}<0.005)$. In addition, lesions in the BAF group tended to be more common as multiple lobar bronchi $(85.0 \%)$ or bilateral bronchi $(73.7 \%)$ than those in the EBLC group.

Radiological characteristics of bronchial trees (such as bronchial stenosis, bronchial wall thickening, atelectasis, peribronchial calcification, and pleural calcification) and lymph nodes (such as lymph node enlargement and location, number, and calcification of lymph nodes) are summarized in Table 4.

Bronchial wall thickening $(25.1 \%$ vs. $6.8 \%, \mathrm{p}<0.05)$ and peribronchial calcification ( $10.2 \%$ vs. $2.6 \%, \mathrm{p}=0.014$ ) were more commonly detected in the BAF group. Although bronchial stenosis was commonly founded in both groups, the detection rate of bronchial narrowing was higher in the BAF group than in the EBLC group (78.6\% vs. $49.1 \%$, p<0.05). Lymph node

Table 1. Baseline characteristics of patients with BAF or EBLC

\begin{tabular}{|c|c|c|c|}
\hline Variable & BAF $(n=167)$ & $\operatorname{EBLC}(n=117)$ & p-value \\
\hline Age, yr & $76.7 \pm 8.1$ & $68.2 \pm 10.2$ & $<0.001$ \\
\hline Female sex & $113(67.7)$ & $18(15.4)$ & $<0.001$ \\
\hline $\mathrm{BMI}, \mathrm{kg} / \mathrm{m}^{2}$ & $21.46 \pm 3.73$ & $21.81 \pm 3.59$ & 0.443 \\
\hline \multicolumn{4}{|l|}{ Smoking } \\
\hline Non-smoker & $148(88.6)$ & $21(17.9)$ & $<0.001$ \\
\hline Ever smoker & $19(11.4)$ & $96(82.1)$ & $<0.001$ \\
\hline Pack years (among ever smoker) & $4.08 \pm 13.81$ & $35.45 \pm 23.52$ & $<0.001$ \\
\hline \multicolumn{4}{|l|}{ Comorbidities } \\
\hline COPD & $16(9.6)$ & $10(8.5)$ & 0.766 \\
\hline Active TB & $64(38.3)$ & $7(6.0)$ & $<0.001$ \\
\hline History of TB & $26(15.6)$ & $17(14.5)$ & 0.810 \\
\hline Diabetes & $30(18.0)$ & $25(21.4)$ & 0.475 \\
\hline Hypertension & $72(43.1)$ & $40(34.2)$ & 0.103 \\
\hline Asthma & $12(7.2)$ & $3(2.6)$ & 0.087 \\
\hline Heart disease & $23(13.8)$ & $9(7.7)$ & 0.483 \\
\hline \multicolumn{4}{|l|}{ Pulmonary function test } \\
\hline $\mathrm{FEV}_{1}, \%$ predicted & $97.9 \pm 26.2$ & $77.3 \pm 21.6$ & $<0.001$ \\
\hline FVC, \% predicted & $91.48 \pm 21.16$ & $81.78 \pm 20.97$ & 0.001 \\
\hline $\mathrm{FEV}_{1} / \mathrm{FVC}$ ratio & $69.96 \pm 14.41$ & $66.65 \pm 14.02$ & 0.115 \\
\hline DLCO & $89.42 \pm 33.56$ & $86.94 \pm 27.56$ & 0.628 \\
\hline
\end{tabular}

Values are presented as mean \pm standard deviation or number (\%).

BAF: bronchial anthracofibrosis; EBLC: endobronchial lung cancer; BMI: body mass index; COPD: chronic obstructive pulmonary disease; TB: tuberculosis; $\mathrm{FEV}_{1}$ : forced expiratory volume in 1 second; FVC: forced vital capacity; DLCO: diffusing capacity of the lung for CO. 
Table 2. Common symptoms of BAF and EBLC

\begin{tabular}{|lccc|}
\hline \multicolumn{1}{|c|}{ Variable } & BAF $(\mathbf{n = 1 6 7 )}$ & EBLC (n=117) & p-value \\
\hline Cough & $77(46.1)$ & $73(62.4)$ & 0.007 \\
Sputum & $63(37.7)$ & $45(38.5)$ & 0.900 \\
Blood-tinged sputum & $4(2.4)$ & $18(15.4)$ & $<0.001$ \\
Dyspnea & $38(22.8)$ & $32(27.4)$ & 0.376 \\
Chest pain & $5(3.0)$ & $14(12.0)$ & 0.014 \\
Fever & $6(3.6)$ & $1(0.9)$ & 0.141 \\
Weight loss & $9(5.4)$ & $13(11.1)$ & 0.078 \\
Others & $31(18.6)$ & $10(8.5)$ & 0.018 \\
\hline
\end{tabular}

Values are presented as number (\%).

BAF: bronchial anthracofibrosis; EBLC: endobronchial lung cancer.

Table 3. Distribution of BAF and EBLC

\begin{tabular}{|lccc|}
\hline \multicolumn{1}{|c}{ Location } & BAF (n=167) & EBLC (n=117) & p-value \\
\hline Right main bronchus & $44(26.4)$ & $21(17.9)$ & 0.047 \\
\hline RUL & $80(47.9)$ & $44(37.6)$ & 0.040 \\
\hline BI & $22(13.2)$ & $0(0)$ & $<0.001$ \\
RML & $90(53.9)$ & $12(12.0)$ & 0.047 \\
\hline RLL superior segment & $20(12.0)$ & $11(9.4)$ & 0.493 \\
RLL & $16(9.6)$ & $27(23.1)$ & 0.002 \\
Left main bronchus & $3(1.8)$ & $18(15.4)$ & $<0.001$ \\
LUL upper division & $68(40.7)$ & $33(28.3)$ & 0.037 \\
\hline LUL lingular division & $26(15.6)$ & $27(23.1)$ & 0.168 \\
LLL superior segment & $25(15.0)$ & $5(4.3)$ & 0.004 \\
LLL & $16(9.6)$ & $15(12.8)$ & 0.389 \\
Multiple & $142(85.0)$ & $46(39.3)$ & $<0.001$ \\
\hline Bilateral & $123(73.7)$ & $8(6.8)$ & $<0.001$ \\
\end{tabular}

Values are presented as number (\%).

BAF: bronchial anthracofibrosis; EBLC: endobronchial lung cancer; RUL: right upper lobe bronchus; BI: bronchus intermedius; RML: right middle lobe; RLL: right lower lobe bronchus; LUL upper: left upper lobe upper bronchus; LUL lingular: left upper lobe lingular bronchus; LLL: left lower lobe bronchus.

enlargements were common in both groups (59.9\% vs. $63.2 \%$, $\mathrm{p}=0.566$ ). Calcified lymph nodes were observed in 61 patients (36.5\%) with BAF, much higher than that in the EBLC group (19 patients, $15.0 \%$ ). Calcified lymph nodes involving more than two sites were more commonly found in the BAF group. After reviewing detailed characteristics, calcified lymph nodes larger than $1.0 \mathrm{~cm}$, focally calcified lymph nodes, and lymph nodes with the extent of calcification less than $50 \%$ were found to be more common in the BAF group than in the EBLC group. Figure 2 shows representative cases of BAF and EBLC.

Biopsy was performed for 17 cases $(10.2 \%)$ in the BAF group and 117 cases $(100 \%)$ in the EBLC group. Pathologic findings were as follows: TB $(n=7,41.2 \%)$, chronic inflammation $(n=5$,
29.4\%), and non-neoplastic bronchial epithelium ( $\mathrm{n}=5,29.4 \%)$ in the BAF group; squamous cell carcinoma ( $n=65,55.6 \%)$, adenocarcinoma $(n=14,12.0 \%)$, small cell lung cancer $(n=22$, $18.8 \%)$, large cell carcinomas $(n=1,0.9 \%)$, both squamous and adenocarcinoma $(n=1,0.9 \%)$, both squamous and small cell carcinoma $(n=1,0.9 \%)$, and others $(n=13,11.0 \%)$ in the EBLC group. Lung cancer stages at diagnosis were as follows: for non-small cell lung cancer, stage I in 17 cases (17.9\%), stage II in 13 cases (13.7\%), stage III in 28 cases (29.5\%), and stage IV in 37 cases (38.9\%); for small cell lung cancer, limited stage in 12 cases $(54.5 \%)$, and extended stage in 10 cases $(45.5 \%)$. 
Table 4. Radiographic characteristics based on CT findings in patients with BAF or EBLC

\begin{tabular}{|c|c|c|c|}
\hline Radiographic characteristics & Patients with BAF $(n=167)$ & Patients with EBLC $(\mathrm{n}=117)$ & p-value \\
\hline \multicolumn{4}{|l|}{ Bronchial tree } \\
\hline Stenosis & $83(49.1)$ & $92(78.6)$ & $<0.001$ \\
\hline Atelectasis & $49(29.3)$ & $29(24.8)$ & 0.397 \\
\hline Bronchial wall thickening & $42(25.1)$ & $8(6.8)$ & $<0.001$ \\
\hline Peribronchial calcification & $17(10.2)$ & $3(2.6)$ & 0.014 \\
\hline Pleural calcification & $2(1.2)$ & $2(1.7)$ & 0.719 \\
\hline \multicolumn{4}{|l|}{ Lymph node } \\
\hline No. of patients with LN enlargement ( $\mathrm{n}=118)$ & $100(59.9)$ & $74(63.2)$ & 0.566 \\
\hline No. of patients with calcified LN (n=80) & $61(36.5)$ & $17(14.5)$ & $<0.001$ \\
\hline 0 & $106(63.5)$ & $100(85.5)$ & \\
\hline 1 & $26(15.6)$ & $9(7.7)$ & \\
\hline$\geq 2$ & $35(21.0)$ & $8(6.8)$ & \\
\hline Size of calcified LN, cm & & & $<0.001$ \\
\hline$\leq 0.5$ & $6(3.6)$ & $2(1.7)$ & \\
\hline$>0.5$ and $\leq 1.0$ & $20(12.0)$ & $6(5.1)$ & \\
\hline$>1.0$ & $35(21.0)$ & $10(7.8)$ & \\
\hline Extent of calcification, $\%$ & & & $<0.001$ \\
\hline$<50$ & 39 (23.4) & $11(9.4)$ & \\
\hline $50-90$ & $14(8.4)$ & $2(1.8)$ & \\
\hline$>90$ & $8(4.8)$ & $4(3.5)$ & \\
\hline Pattern of calcification & & & $<0.001$ \\
\hline Focal & $50(29.9)$ & $11(9.4)$ & \\
\hline Capsular & $4(2.4)$ & $1(0.8)$ & \\
\hline Complete & $7(4.2)$ & $5(4.4)$ & \\
\hline
\end{tabular}

Values are presented as number (\%).

CT: computed tomography; BAF: bronchial anthracofibrosis; EBLC: endobronchial lung cancer; LN: lymph node.

\section{Discussion}

BAF can occasionally mimic other respiratory diseases such as lung cancer and TB. Therefore, it is important for clinicians to know specific characteristics of BAF to make a proper differential diagnosis and rule out possible conditions. In this study, we evaluated differences in clinical and radiological findings between BAF and EBLC. It was found that BAF occurred predominantly in older non-smoking female individuals, with a higher chance of a history of TB than in patients with EBLC. In comparison, the EBLC group was younger with a higher rate of male-smokers than the BAF group. These findings are consistent with previous studies ${ }^{6,7}$. In one study ${ }^{7}$, among BAF patients with a long-term biomass exposure, $80 \%$ of them were females with a mean age of 72 years and most patients (78\%) were non-smokers. In the present study, common symptoms were cough, sputum, and dyspnea in both groups. Although clinical symptoms were not specific, blood-tinged sputum, weight loss, and chest pain were noted more frequently in the EBLC group.

Previous studies have also shown an association between $\mathrm{BAF}$ and $\mathrm{TB}^{1,8-10}$, consistent with our research. In the present study, the rate of patients with coexistent TB was $38.3 \%$. In addition, $15.6 \%$ of patients had a history of TB. In pathologic findings, TB and chronic inflammation occurred in nearly $70 \%$ of patients in the BAF group. Meanwhile, in the EBLC group, the rate of coexistent TB was only $6.0 \%$ and the rate of EBLC patients with a history of TB was $14.5 \%$.

Because the coexistence of pulmonary $\mathrm{TB}$ and lung cancer has been estimated to occur in only about $2 \%$ of cases $^{11}$, BAF can be a highly probable diagnosis compared to EBLC in cases of coexistent active TB.

In pulmonary function test, many reports have stated that BAF is significantly associated with obstructive pattern of airway dysfunction ${ }^{6,7,12,13}$. In our study, the BAF group did not show a substantial decrease of $\mathrm{FEV}_{1}$. This finding might be 


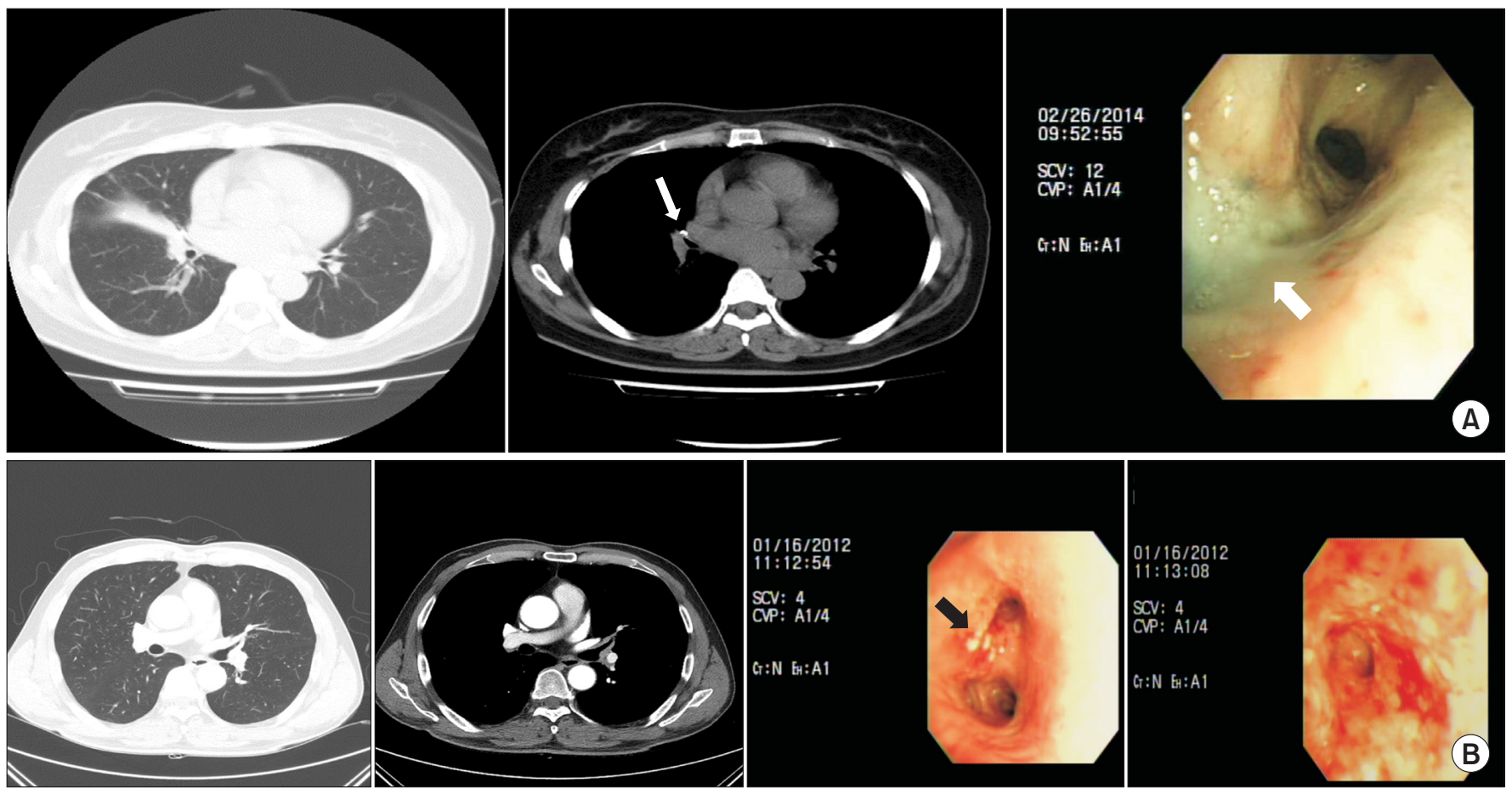

Figure 2. Images showing representative cases of bronchial anthracofibrosis and endobronchial lung cancer. (A) Chest computed tomography (CT) and bronchoscopy of a 70-year-old woman with bronchial anthracofibrosis. Chest CT revealed focal luminal narrowing, atelectasis, and peribronchial calcification (thin white arrow) at the right middle lobar bronchus orifice. The bronchoscopy revealed obliteration of the right middle lobe bronchial orifice due to anthracotic fibrosis (thick white arrow). Bronchoscopic biopsy was not performed. (B) Chest CT and bronchoscopy in a 70-year-old man with bronchial endobronchial lung cancer. CT image showed bronchial wall thickening and luminal irregularity in the left upper lobar bronchus and segmental bronchus. Bronchoscopy showed hypervascular wall nodularity at the left main and the upper lobe bronchi (black arrow). Bronchoscopic biopsy confirmed squamous cell carcinoma.

related to distal small airway dysfunction rather than large airway dysfunction ${ }^{11}$. On the other hand, EBLC patients showed lower values of $\mathrm{FEV}_{1}$ and FVC. Direct airway involvement or external airway compression of lung cancer might have led to a significant airflow obstruction. In addition, space-occupying mass lesion might have reduced compliance of the lung.

In bronchoscopic examination, the BAF group usually involved multiple (85.0\%) or bilateral $(73.7 \%)$ lobar or segmental bronchi, showing upper and middle lobe predominance as reported previously ${ }^{7}$. Upper lobe predominance of BAF tends to be related to TB because the upper lobe is the most commonly affected site of $\mathrm{TB}^{14}$. Meanwhile, involvement of lung cancer is generally unilateral without showing a specific predominance.

In the present study, significant radiologic findings of BAF were bronchial stenosis, atelectasis, bronchial wall thickening, peribronchial calcification, and lymph node calcifications. Although the frequency of different radiologic findings of BAF varied according to studies, stenosis (48\%-100\%), atelectasis (20\%-76\%), bronchial wall thickening (20\%-30\%), and peribronchial calcification (62\%) were common findings, ${ }^{7,13,15-18}$. In our study, bronchial stenosis was the most frequently found lesion in both groups ( $49.1 \%$ vs. $78.6 \%$ ). Rates of peribronchial calcification and bronchial wall thickening were high in the BAF group. Additionally, the rate of patients with lymph node calcification was higher in the BAF group than in the EBLC group. To review detailed characteristics of lymph nodes, calcified lymph nodes involving more than two sites, larger than $1.0 \mathrm{~cm}$, focally calcified lymph nodes, and lymph nodes with the extent of calcification less than $50 \%$ were more common in the BAF group than in the EBLC group. Previous studies have reported that inflammatory necrosis of the lymph node can cause calcium deposit and that lymph node calcification often results from exposure to mineral dust or prior granulomatous infections, especially $\mathrm{TB}^{19,20}$. A higher rate of lymph node calcification in the BAF group might be associated with a higher proportion of TB coexistence. We also investigated the pattern and extent of lymph node calcification because such characteristics of lymph node calcification could made disease more specific. Considering the fact that BAF can arise from exposure of biomass fuels and occupational dust, we anticipated that calcification pattern of BAF could be similar to lymph node pattern of TB or occupational lung disease such as silicosis ${ }^{7,21,22}$. However, eggshell calcification, a clas- 
sic pattern of occupational lung disease, was not detected in this study. Moreover, focal calcification pattern was more common in this study. These findings were inconsistent with results of a previous study showing that complete nodal calcification was the most common finding in $\mathrm{TB}^{23}$. We hypothesized that granular or partially calcified lymph nodes might correspond to the focal pattern category and that these partial dense foci might result from shrinking of inflammatory lesions of relatively large volume lymph nodes in our study group ${ }^{24}$. Metastatic lymph node calcifications can have a granular, amorphous, stippled, or punctate pattern. However, it is difficult to distinguish between benign and malignant diseases depending on the presence or pattern of lymph node calcification $^{25}$. In summary, there were no radiological findings to differentiate between BAF and EBLC precisely. Calcified lymph nodes adjacent to the involved bronchi or peribronchial calcification might be helpful in differentiating this condition from lung cancer. If there are no clinical predisposing factors of BAF, bronchoscopy and biopsy seem to be accurate diagnostic methods. In case of mediastinal lymph node enlargement, which often mimics a metastatic lymphadenopathy in positron emission tomography, endobronchial ultrasound-guided transbronchial needle aspiration can be a more reliable tool for diagnosis ${ }^{26}$.

This study has some limitations. First, it was a small-sized single-center study with a retrospective design. Therefore, the statistical power might be small for findings of the present study. Second, the history of biomass exposure was unavailable. Finally, we used different CT scanners. Despite these limitations, this study described characteristic findings of EBLC and BAF groups. A thorough understanding of clinicradiologic findings of both diseases can help clinicians make a more accurate diagnosis.

In summary, characteristics of BAF include the following: (1) more likely to occur in non-smoking older women, (2) multiple, bilateral involvement with upper and middle lobe predominance, (3) evidence of active TB or a history of TB, and (4) bronchial stenosis, atelectasis, bronchial wall thickening, calcified lymph node, and peribronchial calcification on CT findings. For EBLC, its characteristics are as follows: (1) more likely to occur in male patients with a history of smoking, (2) unilateral involvement without specific predominance, (3) no definite association with TB, and (4) lack of lymph node calcification or peribronchial calcification. Our study demonstrated characteristics of clinical radiologic findings of BAF and EBLC. Increasing awareness of both diseases may help clinicians differentiate them, thus avoiding unnecessary invasive diagnostic and therapeutic procedures.

\section{Authors' Contributions}

Conceptualization: Park TY. Methodology: Yun SY. Formal analysis: Yun SY, Park TY. Data curation: Yun SY. Writing original draft: Yun SY. Writing - review and editing: Yun SY, Park TY. Approval of final manuscript: all authors.

\section{Conflicts of Interest}

No potential conflict of interest relevant to this article was reported.

\section{Funding}

No funding to declare.

\section{References}

1. Chung MP, Lee KS, Han J, Kim H, Rhee CH, Han YC, et al. Bronchial stenosis due to anthracofibrosis. Chest 1998;113: 344-50.

2. Jang SJ, Lee SY, Kim SC, Lee SY, Cho HS, Park KH, et al. Clinical and radiological characteristics of non-tuberculous bronchial anthracofibrosis. Tuberc Respir Dis 2007;63:139-44.

3. Sigari N, Mohammadi S. Anthracosis and anthracofibrosis. Saudi Med J 2009;30:1063-6.

4. Matthews JI, Matarese SL, Carpenter JL. Endobronchial tuberculosis simulating lung cancer. Chest 1984;86:642-4.

5. Smith LS, Schillaci RF, Sarlin RF. Endobronchial tuberculosis: serial fiberoptic bronchoscopy and natural history. Chest 1987;91:644-7.

6. No TM, Kim IS, Kim SW, Park DH, Joeng JK, Ju DW, et al. The clinical investigation for determining the etiology of bronchial anthracofibrosis. Korean J Med 2003;65:665-74.

7. Kim YJ, Jung CY, Shin HW, Lee BK. Biomass smoke induced bronchial anthracofibrosis: presenting features and clinical course. Respir Med 2009;103:757-65.

8. Hwang J, Puttagunta L, Green F, Shimanovsky A, Barrie J, Long R. Bronchial anthracofibrosis and tuberculosis in immigrants to Canada from the Indian subcontinent. Int J Tuberc Lung Dis 2010;14:231-7.

9. Mirsadraee MH, Asnashari AK, Attaran DM. Tuberculosis in patients with anthracosis of lung underlying mechanism or superimposed disease. Iran Red Crescent Med J 2011;13:6703.

10. Mirsadraee M, Saffari A, Sarafraz Yazdi M, Meshkat M. Frequency of tuberculosis in anthracosis of the lung: a systematic review. Arch Iran Med 2013;16:661-4.

11. Kako K, Sakakibara H, Satou M, Suetsugu S. Management of mycobacteriosis in general hospital without isolation ward for tuberculosis patients. 4. Actual status of the management of tuberculosis patients in a university hospital without isolation wards for infectious diseases. Kekkaku 1999;74:145-50. 
12. Jung SW, Kim YJ, Kim GH, Kim MS, Son HS, Kim JC, et al. Ventilatory dynamics according to bronchial stenosis in bronchial anthracofibrosis. Tuberc Respir Dis 2005;59:368-73.

13. Lee HS, Maeng JH, Park PG, Jang JG, Park W, Ryu DS, et al. Clinical features of simple bronchial anthracofibrosis which is not associated with tuberculosis. Tuberc Respir Dis 2002;53:510-8.

14. Fawibe AE, Salami AK, Oluboyo PO, Desalu OO, Odeigha LO. Profile and outcome of unilateral tuberculous lung destruction in Ilorin, Nigeria. West Afr J Med 2011;30:130-5.

15. Kim HY, Im JG, Goo JM, Kim JY, Han SK, Lee JK, et al. Bronchial anthracofibrosis (inflammatory bronchial stenosis with anthracotic pigmentation): CT findings. AJR Am J Roentgenol 2000;174:523-7.

16. Mirsadraee M, Asna-Ashari A, Attaran D, Naghibi S, Mirsadraee S. Bronchial anthracosis: a new diagnosis for benign mass lesions of the lung. Tanaffos 2013;12:10-8.

17. Han FF, Yang TY, Song L, Zhang Y, Li HM, Guan WB, et al. Clinical and pathological features and imaging manifestations of bronchial anthracofibrosis: the findings in 15 patients. Chin Med J (Engl) 2013;126:2641-6.

18. Kim MH, Lee HY, Nam KH, Lim JM, Jung BH, Ryu DS. The clinical significance of bronchial anthracofibrosis associated with coal workers' pneumoconiosis. Tuberc Respir Dis 2010;68:67-73.
19. Storer J, Smith RC. The calcified hilar node: its significance and management: a review. Am Rev Respir Dis 1960;81:85867.

20. Price LW. The pathology of lymph node enlargement. Postgrad Med J 1947;23:401-25.

21. Ooi CG, Khong PL, Cheng RS, Tan B, Tsang F, Lee I, et al. The relationship between mediastinal lymph node attenuation with parenchymal lung parameters in silicosis. Int J Tuberc Lung Dis 2003;7:1199-206.

22. Marchiori E, Hochhegger B, Zanetti G. Lymph node calcifications. J Bras Pneumol 2018;44:83.

23. Gawne-Cain ML, Hansell DM. The pattern and distribution of calcified mediastinal lymph nodes in sarcoidosis and tuberculosis: a CT study. Clin Radiol 1996;51:263-7.

24. Weber AL, Bird KT, Janower ML. Primary tuberculosis in childhood with particular emphasis o hanges affecting the tracheobronchial tree. Am J Roentgenol Radium Ther Nucl Med 1968;103:123-32.

25. Eisenkraft BL, Som PM. The spectrum of benign and malignant etiologies of cervical node calcification. AJR Am J Roentgenol 1999;172:1433-7.

26. Kim MA, Lee JC, Choi CM. Bronchial anthracofibrosis and macroscopic tissue pigmentation on EBUS-TBNA predict a low probability of metastatic lymphadenopathy in Korean lung cancer patients. J Korean Med Sci 2013;28:383-7. 